\title{
The Importance of Histopathological Examination for Safety in Heart Valve Transplantation - Evaluation of Histopathological Findings in Heart Tissues from Valve Donors
}

\author{
Wee Ling Heng ${ }^{1 *}$, Siang Hui Lai ${ }^{2}$, Yeong Phang Lim¹ and Chong Hee Lim ${ }^{1}$ \\ ${ }^{1}$ National Cardiovascular Homograft Bank, Department of Cardiothoracic Surgery, National Heart Centre Singapore \\ ${ }^{2}$ Department of Pathology, Singapore General Hospital, Singapore
}

\begin{abstract}
Most, though not all, heart valve banks performed routine histopathological examination of heart tissues after valve donation. Histopathological examination will enhance the safety of heart valve homografts for transplantation. This is highlighted by a milestone case of cardiac sarcoidosis, a potentially fatal condition which can involve valve leaflet, in a live donor. Since then, testing is mandated for all our bank's donors, with the objective of detecting any cardiac-related contraindications, which might be missed during donor screening. It is also a valuable tool in providing additional information about the cardiac cause of death in deceased donors, especially those who passed away suddenly or from unknown cause. A review of histopathological findings in our donors' heart tissues revealed numerous pathological features despite the small sample size $(n=50)$. Of the deceased donors, $62.9 \%$ were diagnosed with pathological features, among which $72.7 \%$ had multiple abnormalities. $30 \%$ of our bank's donors were heart recipients, who had multiple cardiac abnormalities. Some pathological findings were found to be interrelated. A donor heart with cardiomegaly revealed no pathological features, emphasising the necessity to correlate pathological results with clinical data collated during donor screening for a comprehensive clinical picture in the determination of tissue suitability.
\end{abstract}

Keywords: Cardiac valve donation; Heart; Pathology; Tissue donation; Transplantation

\section{Introduction}

Heart valve homograft remains the preferred graft for children and females of child-bearing age, although its usage accounts for merely a small proportion of heart valve implants in adults [1]. They are especially efficacious for valve replacement in endocarditis and for adult congenital heart patients, who previously underwent valve replacement in childhood. Despite advances in bioprosthetic and mechanical substitutes, the other advantages of homograft which favour its usage includes superior haemodynamic performance over most stented bioprostheses, low incidence of thromboembolic complication, greater resistance to infection and no requirement for anti-coagulation [2].

Currently, the major limitation of homograft use is its limited availability for transplantation, in particular the small valve homografts required for paediatric cases. To expand the donor pool, the National Cardiovascular Homograft Bank (NCHB) recovered heart valves not only from deceased donors, but from live donors, who are the heart recipients as well. When hearts valves are procured from heart recipients, histopathological examination of heart tissue becomes crucial. This is because this group of unique donors has severe heart conditions which might affect the competence and safety of their valves.

The objective of this article is to present a significant case study of a live donor who was diagnosed with cardiac sarcoidosis, and its implication in improving the evaluation process of heart valve suitability in our bank. A review of pathological findings in the heart tissues of all NCHB donors is also presented.

\section{Method}

During the first year of NCHB operations in 2008, histopathological examination was performed only on explanted native remnant hearts from live donors. This was a routine procedure for heart transplantation. National Heart Centre Singapore's (NHCS) heart transplant team would dispatch remnant hearts preserved in $10 \%$ buffered formalin to the Singapore General Hospital (SGH) Department of Pathology for histopathological examination. Gross examination of the remnant hearts were conducted, which included documentation of its size, the weights and lengths of individual fragments, evaluation of cardiac chambers, the remaining heart valves, myocardium and coronary arteries. For microscopic examination, tissues were dissected at 5 microns for histological sections and routinely stained with hematoxylin and eosin, Masson's Trichrome for connective tissue and van Gieson technique for elastic tissue. The sections were examined by the second author.

From year 2009 onwards, fragments of the heart were routinely dispatched for detailed evaluation by the second author. Prior to the separation of aortic and pulmonary valves in the NCHB processing laboratory, tissue specimens of the left and right atrial and ventricular myocardium, aorta, and occasionally pulmonary and coronary arteries were dissected. The tissues were either directly preserved in the $10 \%$ buffered formalin or placed onto a histosette, with full-thickness sections facing upwards, before immersion in formalin. These specimens subsequently underwent wax embedment. Histological sections were at 5 microns and routinely stained with hematoxylin and eosin, Masson's Trichrome and elastic van Gieson stains. The sections were evaluated

*Corresponding author: Heng Wee Ling, National Cardiovascular Homograft Bank National Heart Centre, Singapore, Mistri Wing, 17 Third Hospital Avenue, Singapore 168752, Tel: 6436 7577; Fax : 6221 0019; E-mail: heng.wee.ling@nhcs.com.sg

Received January 17, 2014; Accepted January 28, 2014; Published January 30, 2014

Citation: Heng WL, Lai SH, Lim YP, Lim CH (2014) The Importance of Histopathological Examination for Safety in Heart Valve Transplantation Evaluation of Histopathological Findings in Heart Tissues from Valve Donors. J Cytol Histol S4: 003. doi:10.4172/2157-7099.S4-003

Copyright: $\odot 2014$ Heng WL, et al. This is an open-access article distributed under the terms of the Creative Commons Attribution License, which permits unrestricted use, distribution, and reproduction in any medium, provided the original author and source are credited. 
Citation: Heng WL, Lai SH, Lim YP, Lim CH (2014) The Importance of Histopathological Examination for Safety in Heart Valve Transplantation Evaluation of Histopathological Findings in Heart Tissues from Valve Donors. J Cytol Histol S4: 003. doi:10.4172/2157-7099.S4-003

Page 2 of 4

for the presence of pathological conditions such as cystic medial degeneration or laminar necrosis of the aorta, myocardial ischaemia, muscular degeneration and cardiomyopathy, the presence and extent of cellular inflammation and other clinically significant pathological features. A brief summary of the medical history of the donor was also submitted for review and appropriate clinical-pathological correlation.

\section{Results}

In the first year of operations, $\mathrm{NCHB}$ was not required to perform histopathological examination on the hearts of deceased donors. A milestone in further enhancing the safety of homografts occurred after a routine histopathological examination of a remnant heart from a live donor revealed granulomatous myocarditis with features suggestive of cardiac sarcoidosis. The diagnosis of this autoimmune disease was favoured against that of giant cell myocarditis due to the transmural involvement and vasculitic process in conjunction with well-formed granulomas, a prominent T-cell infiltrate and notable absence of eosinophils within the inflammatory infiltrate. Previously, the donor was diagnosed with idiopathic cardiomyopathy and had multiple episodes of congestive cardiac failure. This contraindication discovered in the post-processing phase subsequently led to a discard of the valves. After the discovery of this contraindication in a donor, NCHB implemented mandatory histopathological examination for all valve donors.

Histopathological findings presented in Table 1 originated from deceased donors between 1.5-65 years old. Only $37.1 \%$ of the heart specimens from deceased (brain dead and cardiac death) donors had normal heart structure upon gross examination with no significant pathological finding. $8.6 \%$ of deceased donors had abnormal enlargement of the heart, a condition known as cardiomegaly detected by macroscopic examination. A majority of $62.9 \%$ were diagnosed with cardiac abnormalities, among which $72.7 \%$ had multiple abnormalities. Of the donors with cardiac abnormalities, $54.5 \%$ had myocyte hypertrophy. $36.4 \%$ had either mild atheroma or coronary atherosclerosis, in contrast to live donors who usually had severe atherosclerosis. While these donors were developing risk factors which might lead to the onset of heart problems, some donors already had pre-existing heart diseases: $18.2 \%$ had cardiac fibrosis. 9.1\% had either primary or hypertrophic cardiomyopathy, which were hereditary cardiac diseases as a result of genetic mutation. $4.5 \%$ had features suggestive of cystic medial degeneration of the aorta. Contraction band necrosis was observed in another donor heart, probably due to the traumatic events leading to her demise rather than a pre-existing heart condition.

From Table 2, the results from live donors aged between 35-59 years

\begin{tabular}{|l|c|}
\hline Types of Pathological Findings & $\begin{array}{c}\text { Number } \\
(\mathbf{N = 3 5 )}\end{array}$ \\
\hline Donor heart with no significant pathological finding & $\mathbf{1 3}$ \\
\hline Donor heart with significant pathological findings & $\mathbf{2 2}$ \\
\hline Significant cardiac abnormalities that the 22 hearts was diagnosed: & \\
\hline Myocyte hypertrophy & 12 \\
\hline Atheroma on aortic wall/Coronary atherosclerosis & 8 \\
\hline Cardiac fibrosis & 4 \\
\hline Cystic medial degeneration in aorta & 1 \\
\hline Primary cardiomyopathy & 1 \\
\hline Hypertrophic cardiomyopathy & 1 \\
\hline Contraction band necrosis & 1 \\
\hline
\end{tabular}

Table 1: Significant cardiac abnormalities in deceased donors.

\begin{tabular}{|l|c|}
\hline Types of Pathological Findings & Number $\mathbf{( N = 1 5 )}$ \\
\hline Severe coronary atherosclerosis & 8 \\
\hline Ischaemic heart disease & 3 \\
\hline Dilated cardiomyopathy & 4 \\
\hline Cardiac fibrosis & 4 \\
\hline Chronic ischaemic cardiomyopathy & 2 \\
\hline Allograft vasculopathy & 2 \\
\hline Post-myocarditic cardiomyopathy & 1 \\
\hline Myxoid degeneration of mitral valves & 1 \\
\hline Cardiac sarcoidosis ('Contraindication) & 1 \\
\hline
\end{tabular}

Table 2: Significant cardiac abnormalities in live donors.

were presented. $30 \%$ of heart valve donors were live donors. All of them had multiple cardiac abnormalities, among which a majority of $60 \%$ had cardiomegaly upon macroscopic examination. Pathological findings discovered included various kinds of cardiomyopathies in $46.7 \%$ of the live donors, ischaemic heart disease in $20 \%$ and cardiac sarcoidosis in $6.6 \% .26 .7 \%$ of the donors developed cardiac fibrosis as a result of myocardial ischaemia. One of the donor hearts became so extensively fibrotic in the epicardium due to high-grade cellular rejection of his previous transplanted heart, which upon gross examination at the recovery site, it was rejected by the Medical Director. $53.3 \%$ had severe coronary atherosclerosis. Allograft vasculopathy affected $13.3 \%$ of donors. $6.6 \%$ developed myxoid degeneration of mitral valves.

\section{Discussion}

The importance of histopathological examination was highlighted when one of our live donors' remnant hearts was diagnosed with granulomatous myocarditis of cardiac sarcoidosis. This condition would easily have evaded detection if it was present in a deceased donor, as histopathological examination was not mandated for heart tissues of such donors back in 2008. Should this occurred it might lead to major clinical and safety consequence to the recipient as transmission of sarcoidosis from a heart donor to the recipient, though extremely rare, had been reported in a case of orthotopic heart transplantation [3].

Cardiac sarcoidosis is an uncommon but potentially fatal condition of unknown etiology. It has been reported to occur in isolation without evidence of sarcoidosis in other parts of the body. Sarcoid granuloma may occur in any location of the heart, with the most common site of involvement being the myocardium $[4,5]$. However, there had also been reported cases of valve leaflet involvement, leading to regurgitation [5]. Sudden death is the most severe manifestation of cardiac sarcoidosis, which usually causes ventricular arrhythmia or complete heart blockage as a result of extensive myocardial involvement [4]. A study in Japan revealed that of the sarcoidosis-related deaths, $46.9 \%$ was associated to cardiac sarcoidosis. However, clinical diagnosis had only been made in $26.7 \%$ of the cases [6], revealing the disease's variable clinical manifestation [5].

Unlike donors with accumulative disorders such as cardiac amyloidosis, a criterion which resulted in the exclusion of a potential live donor during initial donor assessment, the diagnosis of cardiac sarcoidosis in a live patient is more challenging. This is because endomyocardial biopsy, which is considered a gold standard for its detection, is still a relatively insensitive test yielding low diagnostic rate. While cardiac amyloidosis reveals diffused myocardial involvement throughout the myocardium [7], sarcoid granuloma shows localised or heterogeneous distributions within the myocardium and other parts of the heart $[4,5,7]$. Only $25 \%$ of the patients with cardiomyopathy and clinical diagnosis of sarcoidosis were found to have non- 
Citation: Heng WL, Lai SH, Lim YP, Lim CH (2014) The Importance of Histopathological Examination for Safety in Heart Valve Transplantation Evaluation of Histopathological Findings in Heart Tissues from Valve Donors. J Cytol Histol S4: 003. doi:10.4172/2157-7099.S4-003

caseating granulomas on endomyocardial biopsy [5]. Moreover, its symptoms, such as an abnormal electrocardiogram, arrhythmia, valvular abnormalities and dilated cardiomyopathy were non-specific for its diagnosis $[4,5]$. This was probably the case why the live donor developed cardiomyopathy of unknown etiology, leading to cardiac failure requiring transplantation. It was only after her native heart remnant was comprehensively studied by histopathological procedure that her condition was fully elucidated.

After this experience, we realise the importance of histopathological examination and mandate it to become a routine test for all donors since 2009. The objective is to detect any cardiac-related contraindications, which might otherwise be missed during donor screening. Besides, it is a valuable tool in providing additional information about the cardiac cause of death in deceased donors, especially those who passed away suddenly or from unknown cause. Although our bank had not encountered donor referral of sudden cardiac deaths due to nonatheromatous or undiscernable cause, there is a possibility of donors who has morphologically normal hearts with microscopic pathological features yet to be identified, during initial donor screening. Although some diagnoses might be missed due to removal of valves from remnant hearts, it was shown that valve donation did not significantly impair the outcome of the examination [8]. Usually, macroscopically normal hearts would reveal microscopic abnormalities. For instance, an Italian study reported that among the $28 \%$ of their macroscopically normal hearts examined, histological examination disclosed that $79 \%$ of the hearts contained pathological substrates [9]. In another French study, $10 \%$ of unexplained sudden deaths had pathological evidence of arrhythmogenic right ventricular cardiomyopathy [10]. Histopathological examination might also reveal the presence of other cardiac abnormalities, infections, malignancies, systemic or connective diseases which might be relevant for heart valve transplantation [11].

Many pathological features were discovered despite the small sample size $(\mathrm{n}=50)$. This was a significant finding, which further emphasised the importance of histopathological examination in macroscopically normal hearts [12]. However, as heart valves were determined to be unaffected by most of these cardiac abnormalities and the conditions were unlikely to be transmissible to recipients (with the possible exception of cardiac sarcoidosis), they were not contraindications for valve donation. Nevertheless, they provided important reference to the overall condition and function of the donor hearts.

Some pathological findings were discovered to be interrelated. For instance, the presence of myocyte hypertrophy represents a physiological adaptative response to a rise in blood pressure [13]. Hence, its presence is likely to suggest hypertensive changes in the myocardium, which in turn might be an outcome for coronary atherosclerosis. In another instance, severe coronary atherosclerosis had led to myocyte death, myocardial infarction, subsequent scarring or fibrosis, and finally chronic ischaemia cardiomyopathy. This was presented in a case of a live donor diagnosed with severe coronary atherosclerosis, which progressed to end-stage cardiac failure as a result of chronic ischaemic cardiomyopathy, the end-point to myocardial ischaemia.

Besides coronary atherosclerosis, cardiac fibrosis was also found to be generally associated with myocyte hypertrophy. Fibrosis is an expression of interstitial heart disease. For most cases, the endocardium, epicardium and myocardium were the more common sites of involvement. The abnormal proliferation of cardiac fibroblasts and deposition of fibrillar collagen increased diastolic myocardial stiffness and predisposition to heart failure. Although cardiac hypertrophy is a risk factor to cardiovascular disease and increases cardiovascular mortality [13], the development of fibrosis probably has more clinical significance and represents a progression to diastolic cardiac dysfunction. This is because evidence has revealed that the disease is not caused by the mass of myocardium created by hypertrophied myocytes, but rather by the remaining myocardium that is altered by the accumulation of fibrous tissue [14].

It was also interesting to note that of the three deceased donors' hearts which had cardiomegaly, one revealed no microscopic pathological feature. This might indicate a limitation of our bank's practice, which prevented a complete pathological diagnosis of the heart remnant. This was because local legislation allowed for the recovery of heart valve block, whereas the heart remnants had to be returned to the donor's thoracic cavity. Therefore, only representative tissue specimens could be dispatched for examination, which might be inadequate for detection of all clinical abnormalities. This further emphasised the necessity to correlate histopathological results of the tissues with clinical data collated during donor assessment for a more comprehensive clinical picture in the determination of tissue suitability.

Although most banks routinely performed histopathological examination on heart tissues after valve donation, there are some banks which do not practise it [11]. As histopathological examination will definitely enhance the safety of heart valve homografts for transplantation, we strongly recommend all heart valve banks to consider performing this procedure for the benefit of their recipients.

\section{Acknowledgements}

We are grateful to Ministry of Health Singapore's Health Service Developmen Programme grant in support of this programme.

\section{References}

1. Barron DJ, Khan NE, Jones TJ, Willets RG, Brawn WJ (2010) What tissue bankers should know about the use of allograft heart valves. Cell Tissue Bank 11: 47-55

2. Heng WL, Seck T, Tay CP, Chua A, Song C, et al. (2013) Homograft banking in Singapore: two years of cardiovascular tissue banking in Southeast Asia. Cell Tissue Bank 14: 187-194

3. Burke WM, Keogh A, Maloney PJ, Delprado W, Bryant DH, et al. (1990) Transmission of sarcoidosis via cardiac transplantation. Lancet 336: 1579.

4. Ayyala US, Nair AP, Padilla ML (2008) Cardiac sarcoidosis. Clin Chest Med 29: 493-508, ix.

5. Kim JS, Judson MA, Donnino R, Gold M, Cooper LT Jr, et al. (2009) Cardiac sarcoidosis. Am Heart J 157: 9-21.

6. Iwai K, Tachibana T, Takemura T, Matsui Y, Kitaichi M, et al. (1993) Pathologica studies on sarcoidosis autopsy. I. Epidemiological features of 320 cases in Japan. Acta Pathol Jpn 43: 372-376.

7. Uemura A, Morimoto S, Hiramitsu S, Kato Y, Ito T, et al. (1999) Histologic diagnostic rate of cardiac sarcoidosis: evaluation of endomyocardial biopsies. Am Heart J 138: 299-302.

8. Mackey-Bojack S, Roe S, Titus JL (2007) Review of pathologic findings in remnant hearts following valve donation. J Forensic Sci 52: 692-697.

9. Corrado D, Basso C, Thiene G (2001) Sudden cardiac death in young people with apparently normal heart. Cardiovasc Res 50: 399-408.

10. Tabib A, Loire R, Chalabreysse L, Meyronnet D, Miras A, et al. (2003) Circumstances of death and gross and microscopic observations in a series of 200 cases of sudden death associated with arrhythmogenic right ventricular cardiomyopathy and/or dysplasia. Circulation 108: 3000-3005.

11. Visser L, Holsboer E, Bokhorst AG, van Wijk MJ (2012) The value of autopsy and other histological examinations for the safety of tissue transplantation. Cell Tissue Bank 13: 37-46.

12. Fabre A, Sheppard MN (2006) Sudden adult death syndrome and other nonischaemic causes of sudden cardiac death. Heart 92: 316-320. 
Citation: Heng WL, Lai SH, Lim YP, Lim CH (2014) The Importance of Histopathological Examination for Safety in Heart Valve Transplantation Evaluation of Histopathological Findings in Heart Tissues from Valve Donors. J Cytol Histol S4: 003. doi:10.4172/2157-7099.S4-003

Page 4 of 4

13. Takemoto M, Node K, Nakagami H, Liao Y, Grimm M, et al. (2001) Statins as antioxidant therapy for preventing cardiac myocyte hypertrophy. J Clin Invest 108: $1429-1437$
14. Weber KT, Brilla CG, Janicki JS (1993) Myocardial fibrosis: functional significance and regulatory factors. Cardiovasc Res 27: 341-348.
Submit your next manuscript and get advantages of OMICS Group submissions

Unique features:

- User friendly/feasible website-translation of your paper to 50 world's leading languages

Audio Version of published paper

Digital articles to share and explore

Special features:

- 200 Open Access Journals

15,000 editorial team

21 days rapid review process

Quality and quick editorial, review and publication processing

Indexing at PubMed (partial), Scopus, DOAJ, EBSCO, Index Copernicus and Google Scholar etc

Sharing Option: Social Networking Enabled

Authors, Reviewers and Editors rewarded with online Scientific Credits

Better discount for your subsequent articles

Submit your manuscript at: http://www.omicsonline.org/submission 